\author{
Michaela Tscherne \\ Pädagogische Hochschule Niederösterreich, Campus Baden \\ im Gespräch mit
}

Helmut Soher

Polytechnische Schule, Kottingbrunn

\title{
Zum letzten Mal durchatmen in den Ferien
}

DOI: https://doi.org/10.53349/sv.2021.i1.a67

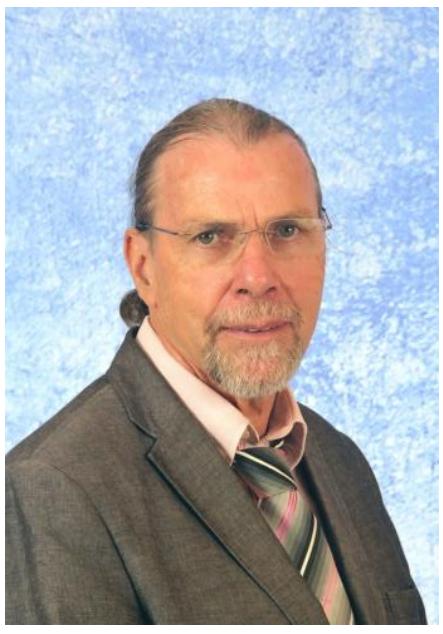

Foto: privat
Helmut Soher, geboren 1956, absolvierte nach seiner Schulzeit in der HTBLA Wiener Neustadt (Hochbau) die Pädagogische Akademie für die Lehrämter Volksschule bzw. Hauptschule in den Fächern Mathematik, Bildnerische Erziehung, evangelische Religion sowie Informatik für das Lehramt Polytechnische Schulen. Die Arbeit mit Jugendlichen war ihm immer schon ein großes Anliegen, so engagierte er sich in der außerschulischen Jugenderziehung, z.B. im Verein Jugendzentren der Stadt Wien, beim Wiener Ferienspiel oder im Verein "Gogomobil" für Kinder und Jugendanimation. Darüber hinaus schloss er eine Ausbildung für den Fachbereich Metall und Elektro sowie zum diplomierten Erziehungsberater und Elterntrainer erfolgreich ab. Seit 1991 ist er an der Polytechnischen Schule Kottingbrunn tätig, zehn Jahre davon als Leiter. Zuvor unterrichtete er an der Hauptschule in Wien 17 und an der PTS Wien 15. Im Herbst 2021 tritt er in den Ruhestand. Anlass für uns, mit ihm über seine letzten Ferien in der Rolle des Schulleiters zu sprechen.

\section{Ein anstrengendes Schuljahr neigt sich dem Ende zu: Welche Zwischenbilanz können Sie nach über einem Jahr Corona in der Schulleitung ziehen?}

Helmut Soher: Sowohl die Schüler*innen als auch wir Lehrer*innen haben im vergangenen Jahr in Bezug auf digitale Kompetenzen viel dazugelernt. Die Bedienung von MS Teams war 
Voraussetzung, um die Herausforderungen der letzten Monate zu bewältigen. Dadurch waren wir gefordert, uns mit diesem Tool näher auseinanderzusetzen, was gut ist. Ich möchte betonen, dass es für die Aufrechterhaltung und die weitere Nutzung Unterstützung braucht. Eine schnelle Internetanbindung sowie neuere, schnellere Geräte wären Voraussetzung dafür, dass wir auch zukünftig digital gut angebunden sind. Obwohl wir gelernt haben, im Distance Learning mit den neuen Werkzeugen umzugehen, muss man schon sagen, dass Präsenzunterricht von größter Bedeutung ist. Das persönliche Gespräch funktioniert im Präsenzunterricht einfach besser. Der Blickkontakt im Zuge von Erziehungsmaßnahmen ist dort wesentlich wirkungsvoller als über die Kamera und den Bildschirm. Auch bei den Lehrer*innenkonferenzen hat uns der persönliche Kontakt sehr gefehlt. Wir haben erkannt, dass die Aufmerksamkeit und die Konzentration im Präsenzunterricht und in den persönlichen Gesprächen besser zu halten sind. Ich denke auch, dass wir bei der Häufigkeit von Konferenzen wieder auf ein gesundes Maß zurückkommen sollten. Mein Leitspruch lautet hier: Weniger ist mehr, auch in der Länge der Konferenzen. Meine Lehrkräfte bekommen vorab die wesentlichen Informationen zur Verfügung gestellt, dadurch können die Besprechungen - seien sie online oder in Präsenz durchgeführt - kürzer gehalten werden.

\section{Wie gelang es Ihnen, in dieser Zeit Energie für sich und das Kollegium freizusetzen?}

Ich habe darauf geachtet, in der Freizeit abzuschalten. Das ist mir zu Hause und in meinem Garten gut gelungen. Was das Kollegium anbelangt, lässt sich diese Frage ganz klar beantworten: mit Ruhe und Gelassenheit. Ich denke, es ist auch sehr wichtig, sich um die Psychohygiene und das Wohlergehen des Kollegiums zu kümmern. Die Lehrkräfte waren in den letzten Monaten nervöser als sonst und sie erleben das Spannungsfeld Schule sehr extrem. Sie sind mit einer bisher nicht bekannten Doppelbelastung konfrontiert, sei es durch eigene Eltern, die gepflegt werden müssen, oder durch ihre Kinder, die im Homeschooling zu betreuen waren oder die vor der Matura standen. Ich versuche, dem Lehrerteam jene Freiheiten zu geben, die es braucht, um ein hohes Maß an Energie in den Unterricht zu stecken. Da war es manchmal schon so, dass die Nerven blank lagen. Fallweise muss der Frust dann herausgelassen werden und für mich hat das bedeutet, dass ich lernen musste, mit diesen Reaktionen umzugehen. Es war wichtig, in solchen Situationen zu erkennen, worum es wirklich geht (und dass es nicht um mich geht). Obwohl mir das manchmal wahnsinnig schwerfiel, habe ich gelernt, mit solchen Situationen umzugehen. Um den Lehrkräften den Rücken zu stärken, habe ich ihnen viele Aufgaben abgenommen. Und ich frage nach, wie es ihnen geht. Plaudern ist da schon ganz wichtig. Als Vorgesetzter sehe ich mich in einer exponierten Position. Einerseits bin ich Vorgesetzter, andererseits möchte ich für meine Lehrer*innen da sein, wenn sie Sorgen und Nöte haben. Daher ist meine Tür auch immer offen für sie. Ich nenne das „seelisches Kümmern“. 


\section{Durchatmen}

\section{Wie fühlt sich Durchatmen mit dem Blick auf die vergangenen Schulleitungsjahre an?}

Die Herausforderungen der letzten Monate waren schon sehr heftig, so gesehen wird mir dieser Stress in meinem zukünftigen Lebensabschnitt nicht wirklich fehlen. Ich habe die Erkenntnis gewonnen, dass man nicht jedem alles recht machen kann. Ich habe immer versucht, den Lehrkräften Freiräume zu schaffen, damit sie ihren Unterricht autonom gestalten können. Vieles ist mir gelungen, anderes weniger, manches gar nicht. Die Bürokratie der letzten Monate war einfach nur mühsam, die Anweisungen und Vorgaben vom Ministerium und der Bildungsdirektion kamen ja meist sehr kurzfristig. Jetzt geht es darum, vorauszuschauen, durchzuatmen. Unter Durchatmen verstehe ich Reflektieren über die letzten Jahre. Momentan komme ich noch nicht dazu, daran zu denken. Jetzt vor dem Schulschluss ist noch viel zu tun, es sind Zeugnisse und Anmeldungen zu schreiben, die Dienstübergabe beginnt noch dieser Tage. Im Augenblick komme ich noch nicht richtig dazu, darüber nachzudenken. Das wird sicherlich noch kommen.

Wie nehmen Sie Ihre Verantwortung dem Kollegium gegenüber wahr, dass auch sie alle immer wieder durchatmen können?

Am wichtigsten erscheint es mir, dass ich für Gespräche zur Verfügung stehe. Ich weise die Lehrkräfte auch immer darauf hin, dass sie mit ihren Kräften gut haushalten sollen. Speziell die neuen Lehrkräfte brauchen ungefähr drei Jahre, bis sie ihren Unterrichtsstil gefunden haben. Zuerst ist man zu streng, dann zu locker, erst im dritten Jahr pendelt man sich dann ein. Manche Lehrkräfte suchen das Gespräch mit mir. Wir haben die jungen Menschen ja nur ein Jahr im Haus, da ist viel zu tun und die Lehrer*innen setzen sich stark unter Druck. Zudem bleibt der Applaus meist aus. Er erreicht uns beispielsweise, wenn die Schüler*innen eine Lehrstelle finden oder nach einigen Jahren zu Besuch kommen und uns erzählen, wie es ihnen ergangen ist. Das sind schon Highlights.

\section{Sie stehen vor einer großen Veränderung. Wie werden Sie in den neuen Lebensabschnitt (durch)starten?}

Ich werde mit Freude in den Ruhestand eintreten und habe große Pläne. Ich habe einen schönen Garten, werde mit Freunden und meiner Partnerin verreisen. Zunächst einmal freue mich auf Ausstellungen, Theater, Kabarett und vor allem auf das Reisen. Ich habe für die nächsten Jahre Reisen nach Mittelamerika, Malta und nach Dubai zur Weltausstellung geplant. Auch der Baikalsee reizt mich sehr. Ich bin Vielreisender, aber es gibt noch genug weiße Flecken auf dem Globus. Ich werde mit Ende Oktober in den Ruhestand gehen. Davor wird im Herbst noch meine Nachfolge eingeschult. Ich habe jetzt schon damit begonnen, viel zu 
delegieren. Ich werde auf alle Fälle auf meine Gesundheit achten, das sehe ich als vordringlich an. Ich plane eine Kur, um meine Verspannungen und meine Rückenschmerzen in den Griff zu bekommen, damit ich gesund in die Pension gehen kann. Der Sommer wird sein wie immer, die Arbeit vor den Ferien wird sicher noch extrem sein, ich werde meine Stellvertreterin einschulen und natürlich auch im Herbst mit Rat und Tat für sie da sein.

\section{Welche drei Empfehlungen können Sie künftigen Schulleiter*innen geben?}

Besonders wichtig scheint es mir zu sein, zu Beginn der Leitertätigkeit nicht alles neu erfinden zu wollen. Sie sollen hervorheben, was gut läuft, und nicht sofort alles verändern. Von Bedeutung ist es auch, den Lehrkräften gut zuzuhören. Die Türe soll sichtbar offen sein für alle und sie sollen oft ins Lehrer*innenzimmer gehen. Es mag nebensächlich erscheinen, aber eine eigene Kaffeemaschine ist keine gute Idee. Holen Sie sich den Kaffee im Lehrerzimmer, bei diesem Anlass ergeben sich gute informelle Gespräche, die das Miteinander fördern. Ausschlaggebend für mich: straight sein, klipp und klar sagen, was man will.

\section{Was können Sie mir noch sagen?}

Genau in sich hineinhören, wenn man das Schulgebäude verlässt. Nichts hinaus mitnehmen. Idealerweise werden alle Aufgaben im Schulgebäude erledigt. Ich habe gelernt, dass ich am Abend, wenn ich rausgehe und die Tür ins Schloss fällt, abschalte. Das kann nur gelingen, wenn ich meine Arbeit nicht mit nach Hause nehme. Es gibt einen Aus-Knopf am Handy: Abschalten im wahrsten Sinne des Wortes. Das war natürlich in Covid-Zeiten schwierig und es ist mir nicht immer gelungen, weil ich das Schultelefon auf das Mobiltelefon umgeleitet habe.

Eines möchte ich noch erwähnen: Die gute Zusammenarbeit mit der Schulaufsicht: Wenn ein*e SQM anruft und einfach nur fragt, wie es mir geht. Das hilft schon und gibt Kraft. Das gehört unbedingt vor den Vorhang.

\section{Mit Helmut Soher, Direktor OSR Ing., sprach}

\section{Michaela Tscherne, Prof. Dr. BEd MBA MSc.}

Professorin und Qualitätsbeauftragte an der Pädagogischen Hochschule Niederösterreich; Arbeits- und Forschungsschwerpunkte: Leadership, Schulautonomie, Personalentwicklung, Berufsorientierung; Publikationen im Bereich Leadership. Kontakt: michaela.tscherne@ph-noe.ac.at 